\title{
Seroprevalence and risk factors for human brucellosis in agro-pastoral areas in Tanzania
}

\author{
Shingo Asakura, ${ }^{1,2}$, George Makingi ${ }^{3}$, Kunda John ${ }^{4}$, Rudovick Kazwala ${ }^{3}$, and Kohei Makita ${ }^{2^{*}}$
}

${ }^{1}$ Ecological Risk Assessment and Control Section, Center for Environmental Biology and Ecosystem, National Institute for Environmental Studies, 16-2, Onogawa, Tsukuba, Ibaraki 305-8506, Japan

${ }^{2}$ Veterinary Epidemiology Unit, School of Veterinary Medicine, Rakuno Gakuen University, 582 Bunkyodai Midorimachi, Ebetsu, Hokkaido 069-8501, Japan

${ }^{3}$ Department of Veterinary Medicine and Public Health, College of Veterinary Medicine and Biomedical Sciences, Sokoine University of Agriculture, P.O.Box 3021, Morogoro, Tanzania

${ }^{4}$ Prime Minister's Office, P.O.Box 3021, 2 Magogoni Street, 11410 Dar es Salaam, Tanzania

\begin{abstract}
Background: Brucellosis is an endemic zoonosis in Tanzania. This study was conducted to investigate the seroprevalence of human brucellosis and its risk factors in agro-pastoral areas in Morogoro Region, Tanzania.
\end{abstract}

Methods: Questionnaire survey and blood sampling were conducted from January to February 2018 at four villages. Anyone living in the villages and wished to participate were involved. Competitive ELISA was used for diagnosis. Risk factor analysis for sero-positivity in human and analysis for the association of sero-positivity between cattle and human within each farm were conducted, using the data of farm-level bovine brucellosis status from our bovine brucellosis research performed in 2016.

Results: The seroprevalence was 33.3\% (44/132). In univariable analysis, the Maasai were significantly more sero-positive (56.5\%) than other tribes (28.4\%) $(\mathrm{OR}=3.23,95 \% \mathrm{Cl}: 1.28-8.41)$. Drinking raw milk was a risk factor in both univariable and multivariable analyses $(\mathrm{OR}=3.97,95 \% \mathrm{Cl}: 1.61-10.20)$. A negative association between sero-positivity in cattle and human within each farm was found $(p<0.01)$. The Maasai performed more risk-taking behaviours for human infection than other tribes: drinking raw milk ( $p<0.01)$ or blood $(p<0.01)$ and helping delivery of cattle with bare hands $(p=0.03)$.

Conclusions: The Maasai were at high risk of human brucellosis. More detailed survey and educational intervention are urgently needed.

Keywords: Human Brucellosis, Risk factors, Agro-pastoral area, Maasai Tanzania

\section{Introduction}

\footnotetext{
*Corresponding Email: kmakita@rakuno.ac.jp
} 
Brucellosis is one of the most prevalent zoonotic diseases in the world caused by bacteria of the genus Brucella. Among six Brucella species based on primary host preference, B. abortus (cattle), B. melitensis (sheep and goats), B. suis (swine) and B. canis (dogs) infections are zoonotic. Human infection occurs through the contacts with infected animals and their tissues, and the consumption of raw dairy products such as unpasteurized milk or cheese. Human brucellosis is a debilitating febrile illness including sweating, malaise, arthralgia and constitutional symptoms, with economical damage due to loss of working time. Since brucellosis diagnosis is hardly performed especially in resource-limited circumstances and the symptoms are non-specific, the disease is often misdiagnosed as other febrile diseases such as malaria and typhoid fever in sub-Saharan African countries. Moreover, the treatment of brucellosis requires long-term costly medication that is not affordable for poor people.

Brucellosis is widely distributed in both humans and animals, with more than 500,000 new cases in human annually all over the world. The disease in both human and livestock has been reported in Tanzania (Shirima \& Kunda, 2016). It is known that occupational risk for human infection is high among farmers, veterinarians, and workers within meat industry. In Tanzania, contact between livestock, wildlife and human is common in pastoral and agro-pastoral areas (Assenga et al., 2015). In addition, risky behaviours for the human infection such as eating raw meat, and drinking raw milk and cattle blood were reported (Asakura et al., 2018b). Thus, rural livestock keeping communities are at high risk of human infection and they should be prioritized in the disease control programs.

Although brucellosis studies have been done in Tanzania, the information about human brucellosis is still limited compared to that on bovine brucellosis, and the risk of the disease transmission from livestock to human is rarely investigated. The objective of this study was to investigate seroprevalence and risk factors for human brucellosis, and the association between bovine and human brucellosis in agro-pastoral areas in Morogoro Region, Tanzania, where brucellosis is endemic both in cattle and human (Asakura et al., 2018a).

\section{Material and methods}

\section{Study site}

Mvomero District is located in the eastern part of Tanzania with around $200 \mathrm{~km}$ from Dar es Salaam which is the country's largest city (Fig. 1). The district's economy strongly depends on agriculture, with crop production and mixed crop-livestock production being main farming systems. The livestock types raised in the district are cattle, goats, sheep, pigs, donkeys, and chickens. Milama, Wami Sokoine, Mvomero and Makuyu villages were selected from the district, where the authors had performed bovine brucellosis research in 2016 prior to the 
current study (Asakura et al., 2018b).

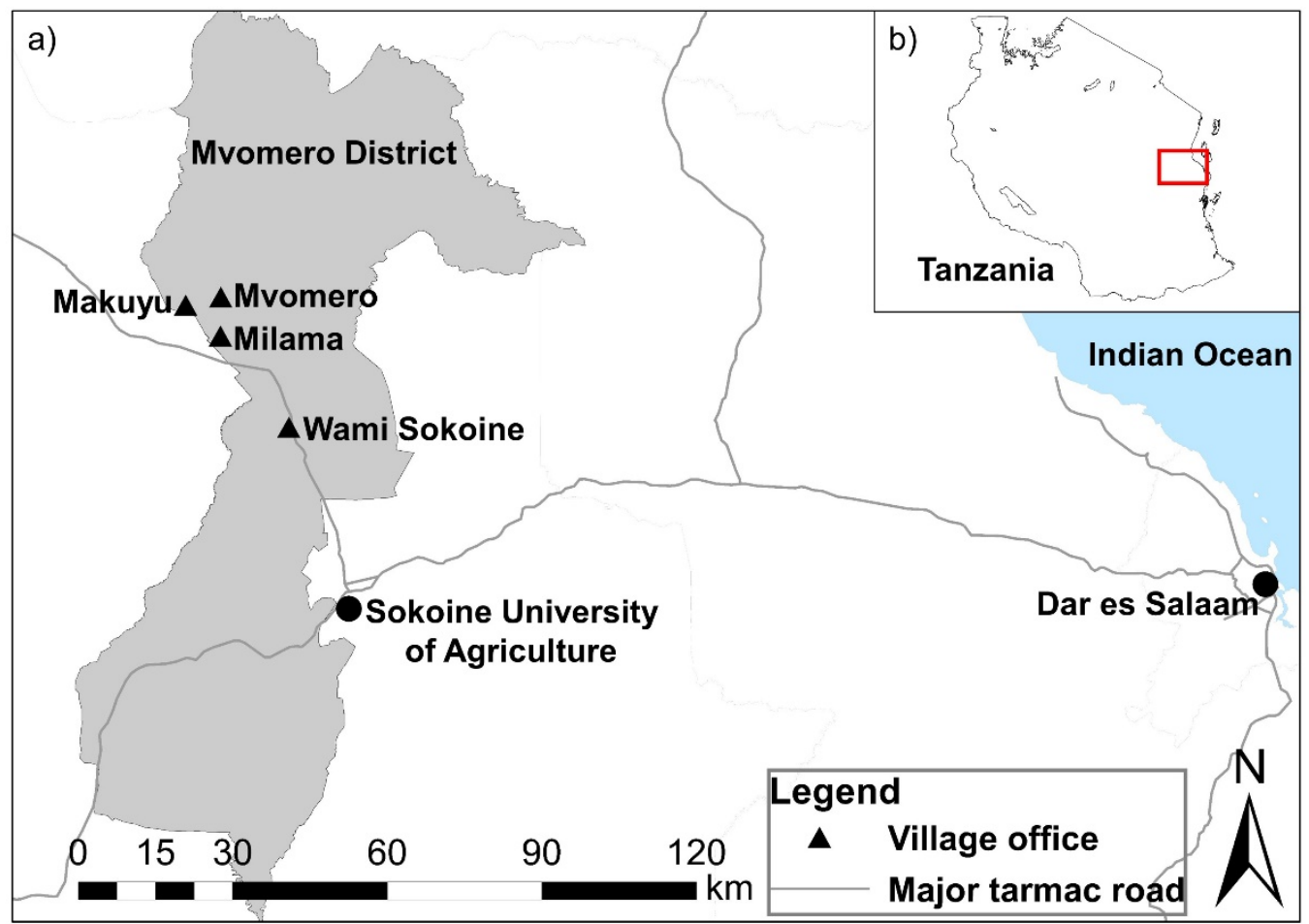

Fig. 1. Map of the study area. a: Mvomero District highlighted in grey showing studied villages; $\mathbf{b}$ : a square shows the location of a within the Republic of Tanzania

\section{Study design, field survey and diagnostic test}

This was a cross-sectional study involving blood sampling and interviews using a structured questionnaire. A field survey was conducted from January to February 2018 in the selected villages. Recruitment process was operated by the agricultural, veterinary and medical officers and the authors, targeting mainly farm owners who had participated in bovine brucellosis research conducted by the authors in 2016 and their family members (Asakura et al., 2018b). As a result, however, anyone who lived in the villages and wished to participate the research were involved. Therefore, sample size was not calculated. Information about participant, the animals kept, knowledge about brucellosis and behaviours associated with human infection was collected using a structured questionnaire which was originally prepared in English and then translated to Swahili, which is a national language in Tanzania. Two $\mathrm{ml}$ of blood was collected in vacutainer serum tubes. Blood samples were left for 1 hour at ambient temperature to separate serum from the blood. Serum samples were stored in a freezer at $-20^{\circ} \mathrm{C}$ at the laboratory in Sokoine University of Agriculture until diagnostic test. Serum samples were tested by competitive enzyme-linked immunosorbent assay (CELISA) 
(Boehringer Ingelheim Svanova, Uppsala, Sweden). CELISA test was performed according to the manufacturer's instructions at Sokoine University of Agriculture. Briefly, sample dilution buffer was added into each well. Then, positive, weak positive and negative serum controls, sample dilution buffer for conjugate control and test samples were added into each of the appropriate wells, respectively. After adding mAb-solution into all wells used for controls and samples, the plate was sealed and the reagents were mixed using a plate shaker. Subsequent to 30 minutes of incubation at room temperature, the plate was rinsed with PBS Tween Buffer and conjugate solution was added into each well. After the same process of incubation and rinse written above, substrate solution was added and incubated for 10 minutes at room temperature. Then, stop solution was added and mixed. The optical density (OD) was measured at $450 \mathrm{~nm}$. The percent inhibition (PI) values for controls as well as samples were calculated using the formula: PI (\%) =100 - (OD [samples or control] $\times 100 /$ OD [conjugate control]). The samples were regarded as negative for Brucella antibodies ( $\mathrm{PI}<30 \%$ ) and positive for Brucella antibodies ( $\mathrm{PI} \geq 30 \%$ ).

\section{Data analysis}

CELISA test prevalence was taken as a true prevalence in the study due to the high sensitivity and high specificity of CELISA which were 1.000 and 0.999 , respectively. For univariable risk factor analysis, associations between Brucella seropositivity and each factor of the questionnaire were analysed. Wilcoxon rank-sum test was performed for count data. Pearson's chi-squared test with Yates' continuity correction was used for binary data, and the odds ratio and the $95 \%$ confidence interval were calculated for the studied variables.

Multivariable analysis was performed using GLM with binomial family, selecting seropositivity as outcome variables, and variables with $p$ values $<0.2$ in univariable analyses as explanatory variables. Backward stepwise selection method with likelihood ratio test was used to obtain a final model.

In addition, farm-level bovine brucellosis status obtained in the previous research by herd-milk indirect ELISA (Asakura et al., 2018b) was used to investigate the association between bovine brucellosis and human brucellosis within each farm. The association of seropositivity between cattle and human within each farm was analysed using Fisher's exact test, and those between conduct of risky behaviours against human infection and tribes focusing on Maasai were done using Pearson's chi-squared test and Fisher's exact test. Fisher's exact test was conducted when at least one cell included an expected frequency of less than 5 . Statistical significance was set at $p$ values $<0.05$. Statistical analyses were performed using the computer software $\mathrm{R}$ version 3.5.1. 


\section{Results}

\section{Characteristics of the participants}

Total 132 people were involved, and 53 were from the 32 farms which participated in the previous bovine brucellosis research (Asakura et al., 2018b). Table 1 shows the characteristics of the participants with the levels of education and knowledge, livestock keeping status, and risky behaviours for human infection. Tribe of 63 participants were not identified because they were unwilling to answer. However, it was confirmed Maasai were not included in the 63 participants.

Table 1. Characteristics of the participants

\begin{tabular}{|c|c|c|}
\hline Categories & Response $(n=132)$ & Percentage \\
\hline Sex: Male & 77 & 58.3 \\
\hline \multicolumn{3}{|l|}{ Age group (years) } \\
\hline $7-20$ & 27 & 20.5 \\
\hline $21-40$ & 45 & 34.1 \\
\hline $41-72$ & 60 & 45.5 \\
\hline \multicolumn{3}{|l|}{ Tribe } \\
\hline Maasai & 23 & 17.4 \\
\hline Mbulu & 11 & 8.3 \\
\hline Zigua & 6 & 4.5 \\
\hline Chaga & 5 & 3.8 \\
\hline Jita & 4 & 3.0 \\
\hline Sukuma & 4 & 3.0 \\
\hline Hehe & 3 & 2.3 \\
\hline Lugulu & 3 & 2.3 \\
\hline Shambaa & 3 & 2.3 \\
\hline Pare & 2 & 1.5 \\
\hline Kaguru & 1 & 0.8 \\
\hline Meru & 1 & 0.8 \\
\hline Muha & 1 & 0.8 \\
\hline Ngindo & 1 & 0.8 \\
\hline Ngoni & 1 & 0.8 \\
\hline Not identified (unwilling to answer) & 63 & 47.7 \\
\hline \multicolumn{3}{|l|}{ Level of education } \\
\hline No education & 19 & 14.4 \\
\hline
\end{tabular}




\begin{tabular}{lll}
\hline Categories & Response $(\mathbf{n}=\mathbf{1 3 2})$ & Percentage \\
\hline Primary & 113 & 85.6 \\
Knowledge about brucellosis & & \\
Name & 50 & 37.9 \\
Symptoms & 28 & 21.2 \\
Transmission from animal to human & 37 & 28.0 \\
Keeping cattle & 64 & 48.5 \\
Keeping small ruminants & 47 & 35.6 \\
Milking & 46 & 34.8 \\
Drinking raw milk & 25 & 18.9 \\
Drinking cattle blood & 17 & 12.9 \\
Helping delivery of cattle with bare hands & 31 & 23.5 \\
\hline
\end{tabular}

\section{Prevalence and risk factors for brucellosis}

The human brucellosis prevalence was $33.3 \%(44 / 132,95 \% \mathrm{Cl}: 25.5-42.1)$. The median age of all participants was 40 (2.5th and 97.5th percentiles: 8-66), and those of positive and negative participants were 39 (2.5th and 97.5th percentiles: 10-65) and 40 (2.5th and 97.5th percentiles: 7-66), respectively, showing no significant difference among them $(p=0.89)$. Table 2 shows the results of univariable analyses for brucellosis for binary data. Significant variables associated with brucellosis were the Maasai tribe $(\mathrm{OR}=3.23,95 \% \mathrm{Cl}: 1.28-8.41)$ and drinking raw milk ( $\mathrm{OR}=3.97,95 \% \mathrm{Cl}: 1.61-10.20)$. The latter, drinking raw milk, was also the single significant variable in the multivariable analysis.

Table 2. Univariable risk factor analysis for binary response variable $(n=132)$.

\begin{tabular}{lllllll}
\hline \multirow{2}{*}{ Factors } & Response & Positive & Negative & Prevalence $(\%)$ & $\begin{array}{l}\text { Odds } \\
\text { ratio }\end{array}$ & $95 \% \mathrm{Cl}$ \\
\hline Sex & Male & 23 & 54 & 29.9 & 0.69 & $0.33-1.45$ \\
& Female & 21 & 34 & 38.2 & & \\
Age group (years) & $7-20$ & 10 & 17 & 37.0 & - & - \\
& $21-40$ & 15 & 30 & 33.3 & 0.85 & $0.31-2.37$ \\
& $41-72$ & 19 & 41 & 31.7 & 0.79 & $0.30-2.11$ \\
Tribe & Maasai & 13 & 10 & 56.5 & 3.23 & $1.28-8.41$ \\
& Others & 31 & 78 & 28.4 & & \\
Education & No & 7 & 12 & 36.8 & 1.21 & $0.41-3.30$
\end{tabular}




\begin{tabular}{|c|c|c|c|c|c|c|}
\hline Factors & Response & Positive & Negative & Prevalence (\%) & $\begin{array}{l}\text { Odds } \\
\text { ratio }\end{array}$ & $95 \% \mathrm{Cl}$ \\
\hline & Primary & 37 & 76 & 32.7 & & \\
\hline \multirow[t]{2}{*}{ Knowledge - Name } & Yes & 16 & 34 & 32.0 & 0.91 & $0.42-1.92$ \\
\hline & No & 28 & 54 & 34.1 & & \\
\hline \multirow[t]{2}{*}{$\begin{array}{l}\text { Knowledge - } \\
\text { Symptoms }\end{array}$} & Yes & 8 & 20 & 28.6 & 0.76 & $0.29-1.87$ \\
\hline & No & 36 & 68 & 34.6 & & \\
\hline \multicolumn{7}{|l|}{ Knowledge - } \\
\hline \multirow{2}{*}{$\begin{array}{l}\text { Transmission from } \\
\text { animal to human }\end{array}$} & Yes & 14 & 23 & 37.8 & 1.32 & $0.58-2.92$ \\
\hline & No & 30 & 65 & 31.6 & & \\
\hline \multirow[t]{2}{*}{ Keeping cattle } & Yes & 21 & 43 & 32.8 & 0.96 & $0.46-1.98$ \\
\hline & No & 23 & 45 & 33.8 & & \\
\hline \multirow[t]{2}{*}{$\begin{array}{l}\text { Keeping small } \\
\text { ruminants }\end{array}$} & Yes & 16 & 31 & 34.0 & 1.05 & $0.49-2.24$ \\
\hline & No & 28 & 57 & 32.9 & & \\
\hline \multirow[t]{2}{*}{ Milking } & Yes & 15 & 31 & 32.6 & 0.95 & $0.44-2.04$ \\
\hline & No & 29 & 57 & 33.7 & & \\
\hline \multirow[t]{2}{*}{ Drinking raw milk } & Yes & 15 & 10 & 60.0 & 3.97 & $1.61-10.20$ \\
\hline & No & 29 & 78 & 27.1 & & \\
\hline \multirow[t]{2}{*}{$\begin{array}{l}\text { Drinking cattle } \\
\text { blood }\end{array}$} & Yes & 8 & 9 & 47.1 & 1.94 & $0.67-5.57$ \\
\hline & No & 36 & 79 & 31.3 & & \\
\hline $\begin{array}{l}\text { Helping delivery of } \\
\text { cattle with bare } \\
\text { hands }\end{array}$ & Yes & 11 & 20 & 35.5 & 1.14 & $0.47-2.64$ \\
\hline & No & 33 & 68 & 32.7 & & \\
\hline
\end{tabular}

\section{Bovine brucellosis and human brucellosis within each farm}

The association between farm-level bovine brucellosis and human brucellosis within each farm was analysed using the data of previous bovine brucellosis research by herd-milk (Asakura et al., 2018b) and 53 participants from the previously studied farms. Significantly negative association was found between the factors: cattle farming families without cattle infected with bovine brucellosis (18/31, 58.1\%) had higher prevalence than the families with infected cattle $(1 / 22,4.5 \%, p<0.01)$. 


\section{Risky behaviours of Maasai and other tribes}

Table 3 shows the results of univariable analysis for behaviours of Maasai and other tribes. Maasai significantly conducted drinking raw milk $(p<0.01)$, drinking cattle blood $(p<0.01)$ and helping delivery of cattle with bare hands $(p=0.03)$ than other tribes.

Table 3. Proportions of Maasai and the other tribe participants conducting risky behaviours for human infections with Brucella

\begin{tabular}{llllll}
\hline Factors & $\begin{array}{l}\text { Maasai } \\
(\mathrm{n}=23)\end{array}$ & Percentage & $\begin{array}{l}\text { Other tribes } \\
(\mathrm{n}=109)\end{array}$ & Percentage & P value \\
\hline Milking & 12 & 52.2 & 34 & 31.2 & 0.09 \\
Drinking raw milk & 12 & 52.2 & 13 & 11.9 & $<0.01$ \\
Drinking cattle blood & 9 & 39.1 & 8 & 7.3 & $<0.01$ \\
Help delivery of cattle & 10 & 43.5 & 21 & 19.3 & 0.03 \\
\hline
\end{tabular}

\section{Discussion}

In the present study, the seroprevalence of human brucellosis in agro-pastoral areas in Mvomero District in Morogoro Region, Tanzania was evaluated by CELISA. A previous study reported that the human brucellosis prevalence was 36.1\% in Mvomero District by RBPT, supporting our result of the endemic situation of human brucellosis in the area (James, 2013). In sub-Saharan countries including Tanzania, human brucellosis information is limited and the quality of the data is poor (Rubach et al., 2013). This study also had limitations in samples due to the use of non-probability sampling method and the lack of sample size calculation. It would be ideal to conduct an additional study with an adequate sampling process and enough sample size based on the calculation to avoid potential sampling bias.

While other studies evaluated the relationships between seroprevalences of brucellosis in humans and livestock in large unit or wide area, this study investigated the association of seropositivity between cattle and human within each farm. Our previous research confirmed the bovine brucellosis endemic situation in the Mvomero District with $7.0 \%$ of animal-level and $44.4 \%$ of herd-level, respectively (Asakura et al., 2018b, Asakura et al., 2018a). In general, brucellosis in human is closely linked with that in animals. However, within each farm-level, this study showed counter-intuitive association on the infections between cattle and human. For the correct causality, the result should be interpreted that no association was found between them. Possible reasons for the result are the limitation of milk sample diagnosis which involved only milking cows during the sampling time, small sample size which might have caused sampling bias, and not having conducted further 
multivariable analysis, and the lack of information about brucellosis infection status in small ruminants. Several studies reported that small ruminants are more likely to be associated with human infection than cattle (Viana et al., 2016). Therefore, although keeping small ruminants was not a risk factor in this study, further information about brucellosis in small ruminants is necessary to understand the dynamics of the disease. The other possible cause is that participants might be infected earlier from their animals, or from raw milk produced by the other farms. Regarding the latter, since drinking raw milk was a significant risk factor for human infection in the current study, further information such as the milk value chain and the distribution area of milk would be important to control human infection.

The Maasai showed higher seroprevalence than other tribes and tended to conduct risky behaviours, including consumption of raw milk. Given the result, they are supposed to use clinics and hospitals due to the fever and other symptoms caused by brucellosis. In fact, however, they rarely appear to medical facilities (personal communication with Mvomero District medical officer). Since the Maasai have their traditional culture, more information about the sociological aspects of them is required.

Knowledge about brucellosis was not enough among the participants. Several studies indicated that knowledge of the transmission routes for brucellosis had a preventive effect for human infection (Sofian et al., 2008). Thus, disease control program should include public health education in the study areas, especially prioritizing Maasai people to change high-risk behaviours for human infection.

In conclusion, the current study is a partially integrated human and animal research with a "One Health" concept, getting cooperation from both the health and livestock sectors. This kind of "One Health" approach including cross-sectoral collaboration should be accelerated for the control of transboundary animal diseases, zoonoses and neglected tropical diseases including brucellosis (Zinsstag et al., 2011). Given high brucellosis prevalence in human in the study area, deeper and wider study, and intervention are urgently needed.

\section{Ethics approval and consent to participate}

This study was approved by the Ethical Committee of the Graduate School of Dairy Science, Rakuno Gakuen University (approval number 17-4) and the Medical Research Coordinating Committee of the National Institute for Medical Research in the Republic of Tanzania (reference number NIMR/HQ/R.8a/Vol. IX/2655). Permission to conduct the study in Mvomero District was sought and granted by district executive officers. Prior to the sampling and questionnaire survey, informed consent was obtained from the adult participants, and for child participants, informed assent and informed consent were obtained from them and their parent or guardian, respectively. 


\section{Funding}

This work was supported by the Ministry of Education, Culture, Sports, Science and Technology of Japan under the research project "Development of rapid diagnostic kits for infectious pathogens in industry animals and establishment of effective control methods through global analysis of transmission routes (grant number S1391001)" of "2013 Support grant for establishment of strategic research platform for private universities".

Declaration of interest: The authors declare that they have no competing interests.

\section{Acknowledgments}

The authors would like to thank all participants in the survey. We also thank Mvomero district officials and veterinary and medical officers for their cooperation during the implementation of this study.

\section{References}

Asakura, S., G. Makingi, R. Kazwala and K. Makita. (2018a) Brucellosis Risk in Urban and Agro-pastoral Areas in Tanzania. EcoHealth 15: 41-51.

Asakura, S., G. Makingi, R. Kazwala and K. Makita. (2018b) Herd-level risk factors associated with Brucella sero-positivity in cattle, and perception and behaviours on the disease control among agro-pastoralists in Tanzania. Acta tropica 187: 99-107.

Assenga, J. A., L. E. Matemba, S. K. Muller, J. J. Malakalinga and R. R. Kazwala. (2015) Epidemiology of Brucella infection in the human, livestock and wildlife interface in the Katavi-Rukwa ecosystem, Tanzania. BMC veterinary research 11: 189.

James, L. W. (2013) Studies of Human Brucellosis in Mikumi-Selous Ecosystem, Morogoro,Tanzania. M.Sc. dissertation, Sokoine University of Agriculture.

Rubach, M. P., J. E. Halliday, S. Cleaveland and J. A. Crump. (2013) Brucellosis in low-income and middleincome countries. Current opinion in infectious diseases 26: 404-412.

Shirima, G. M. and J. S. Kunda. (2016) Prevalence of brucellosis in the human, livestock and wildlife interface areas of Serengeti National Park, Tanzania. The Onderstepoort journal of veterinary research 83: a1032.

Sofian, M., A. Aghakhani, A. A. Velayati, M. Banifazl, A. Eslamifar and A. Ramezani. (2008) Risk factors for human brucellosis in Iran: a case-control study. International journal of infectious diseases 12: $157-161$.

Viana, M., G. M. Shirima, K. S. John, J. Fitzpatrick, R. R. Kazwala, J. J. Buza, S. Cleaveland, D. T. Haydon and J. E. Halliday. (2016) Integrating serological and genetic data to quantify cross-species transmission: brucellosis as a case study. Parasitology 143: 821-834.

Zinsstag, J., E. Schelling, D. Waltner-Toews and M. Tanner. (2011) From "one medicine" to "one health" and systemic approaches to health and well-being. Preventive veterinary medicine 101: 148-156. 